\title{
Nonvolatile Dynamic Memories
}

\author{
Sima Dimitrijev
}

\begin{abstract}
This paper demonstrates that electronically passivated $\mathrm{Si}_{-} \mathrm{SiO}_{2}$ interface enables the development of nonvolatile dynamic memories. Experimental results on chargeretention times are presented to illustrate that the Si DRAMs would become nonvolatile memories if implemented into SiC. The disadvantages of the DRAM cell (1ClT), in terms of limited memory-capacity increase, are discussed to highlight the need for development of superior memory cells.
\end{abstract}

\section{INTRODUCTION: SEMICONDUCTOR MEMORIES BEYOND DRAM AND FLASH}

Dynamic Random-Access Memory (DRAM) and Flash are two types of commercially available siliconbased memories. The memory element in DRAMs is a capacitor that is accessed by a Si-based MOSFET acting as electronic switch. The leakage currents through the $\mathrm{Si}$ MOSFETs are so high that the memory cell has to be refreshed hundreds of times per second. As a consequence the memory draws power contunuosly, even when data is not being read or written, and it loses the data when the power is switched off. DRAM is, however, the only available memory at present that can be used for data processing as it enables unlimited number of fast writing cycles. The other type of commercially available memory - flash - is used for data storage. It is a nonvolatile memory, based on a single-transistor cell with a floating polysilicon gate inserted between the control gate and the MOSFET channel. To write data in flash cells, electrons are forced through the dielectric surrounding the floating gate. This results in relatively long writing times and severely limited number of writing cycles, preventing the use of this memory type for data processing.

Both types of semiconductor memories, DRAM and flash, are approaching their limits in terms of memorycapcity increase. The problem with the $1 \mathrm{ClT}$ cell in DRAMs is that it. is increasingly difficult to sense the charge stored by ever decreasing capacitance that is necessary for the capacity increase. In the case of flash, the limit to the capacity increase is due to high voltages needed to inject electrons into the floating gate. This problem will be alleviated by the use of multiple memory levels, but nonetheless, the capacity increase of flash memories becomes increasingly difficult.

S. Dimitrijev is with the School of Microelectronic Engineering, Griffith University, Queensland, Australia, E-mail: s.dimitrijev@griffith.edu.au.
To address these limitations of DRAM and flash, a number of alternative technologies are being investigated as potential successors of flash and/or DRAM. An excellent review article by Geppert [1] describes the three main contenders: ferroelectric RAM, magnetoresistive RAM, and the Ovonic Unified Memory that is based on phase changes of heated chalcogenide alloys. These are all nonvolatile memories that are being developed by the leading chip manufacturers as potential successors of flash and possibly DRAMs. In spite of this intensive development, however, all these technologies continue to demonstrate serious problems in terms of achieving memory capacities that would approach modern flash and DRAM. It may seem ironic, but the difficulties of these new technologies are exactly with the property that they need to improve to be considered as flash and DRAM alternatives.

Recent successful efforts to electronically passivate $\mathrm{SiC}-\mathrm{SiO}_{2}[2]-[33]$ provide a new opportunity to address the limitations associated with flash and DRAM. This paper describes the potential of $\mathrm{SiC}$ as a memory material.

\section{Charge Retention Times}

A gate voltage that is larger (in absolute terms) than the threshold voltage of a MOS capacitor on $\mathrm{SiC}$ sets the MOS capacitor in deep depletion mode. This is because the thermal generation of minority carriers is extremely low at room temperature and there is no other source that would provide the minority carriers. The depletion layer at the semiconductor surface is then extended beyond the stronginversion value to provide the depletion-layer charge as a replacement for the inversion-layer charge. As a result, the deep depletion capacitance drops below the value of the strong-inversion capacitance.

The deep depletion mode is a nonequilibrium state of the MOS capacitor. This means, the MOS capacitance should increase from the deep depletion level toward the inversion level as the generation mechanisms provide minority carriers that accumulate in the inversion layer. This process is immeasurably slow at room temperature for MOS capacitors with good $\mathrm{SiC}-\mathrm{SiO}_{2}$ interfaces. However, the capacitance change can be measured at temperatures high enough to accelerate the generation rate. Assuming exponential increase of the capacitance from the deep depletion loward the inversion level, the time constant of the exponential dependence can be extracted from the measured results. This time constant is the capacitance relaxation time. However, it also provides a good estimate 
of how long a nonequilibrium charge can be retained at a depleted SiC surface. Therefore, this time constant can also be referred to as charge retention time

Fig. I shows measured charge-retention times for MOS capacitors on $4 \mathrm{H}-$ and $6 \mathrm{H}-\mathrm{SiC}$ with gate oxides obtained by two different nitridation techniques: (1) the sandwich process $\left(100 \% \mathrm{NO} /\right.$ dry $\left.\mathrm{O}_{2} / 100 \% \mathrm{NO}\right)$ and (2) high-temperature diluted- $\mathrm{N}_{2} \mathrm{O}$ process [31].

Assuming Arrhenius type dependence of the chargeretention times on temperature, the high-temperature results can be extrapolated to room temperature. This provides estimates of the charge-retention times at room temperature. In the case of $4 \mathrm{H}-\mathrm{SiC}$, the room temperature retention times were $10^{10}$ years for the sandwich process and $10^{9}$ years for the diluted $\mathrm{N}_{2} \mathrm{O}$ process. The sandwich process on $6 \mathrm{H}-\mathrm{SiC}$ produced a lower value of $10^{6}$ years. As these results illustrate, the charge-retention times are strongly affected by the gate-oxide processing, which indicates a strong dependence on the interface-trap density. This conclusion has been confirmed in a subsequent study of the generation rate [30], showing that the creation of the inversion layer is due to surface generation whereas all other mechanisms (including bulk generation in the depletion layer) are negligible.

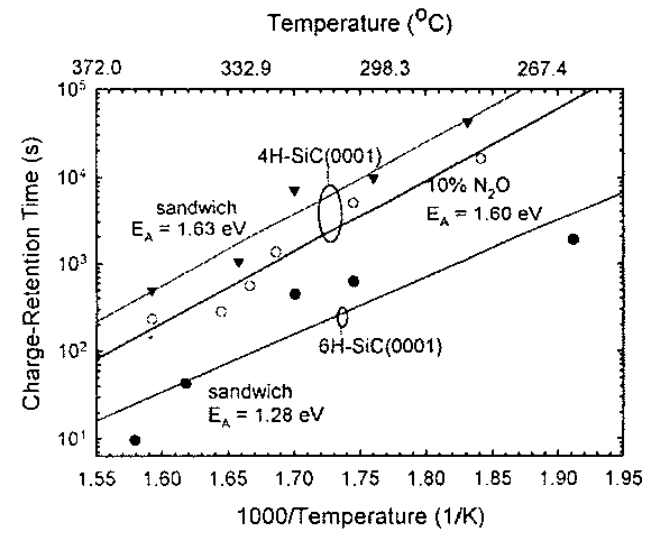

Fig. 1. Arrhenius plots for charge-retention times for MOS capacitors on $4 \mathrm{H}$ - and $6 \mathrm{H}-\mathrm{SiC}$ as a function of temperature for gate oxides grown by different processe: $4 \mathrm{H}-\mathrm{SiC}$, sandwich process, $(\boldsymbol{\nabla}) ; 4 \mathrm{H}-\mathrm{SiC}$, high temperature $10 \% \mathrm{NO}$, $(\mathrm{O}) ; 6 \mathrm{H}-\mathrm{SiC}$, sandwich process, (O).[31]

Importantly, the charge retention times estimated in these experiments are so long that they clearly demonstrate that it is possible to develop nonvolatile memories in $\mathrm{SiC}$.

\section{Nonvolatile Dynamic RAM Based on the 1T/1C CELL}

The silicon-based DRAMs are based on the so called 1T/1C memory cell. Fig. 2 shows the connection of $1 \mathrm{~T} / 1 \mathrm{C}$ cells to form a DRAM array. The capacitors in this array are the memory elements, whereas the MOSFETs are the switches that provide access to the memory elements for information reading and writing. The MOSFETs are off when the gates are not selected (floating) for either information reading or writing. The nonequilibrium state of this memory cell is when the capacitor is charged by positive voltage with respect to the grounded electrode. This means the source-to-bulk junction of the MOSFET is reverse biased to prevent leakage of the nonequilibrium charge. In the silicon implementation, however, the reverse-biased current of the source-to-substrate junction is so high that the capacitor is discharged within tens of milliseconds. Consequently, every cell in the DRAM array has to be periodically refreshed to maintain the information. When the power is switched off, the memory loses the information. Because of this, this type of memory is called dynamic RAM. The word dynamic is used to distinguish this type of memory from the static RAM that is based on cells consisting of flip flops. The static RAM is also volatile in the sense that it loses its information when the power is switched off, but it does not need to be refreshed during the operation. The cell size of the DRAM is much smaller enabling much higher memory capacities compared to SRAM. This factor is so important that it is the DRAM that dominates the memory market for data processing, regardless of the disadvantage related to the need to periodically refresh the memory.

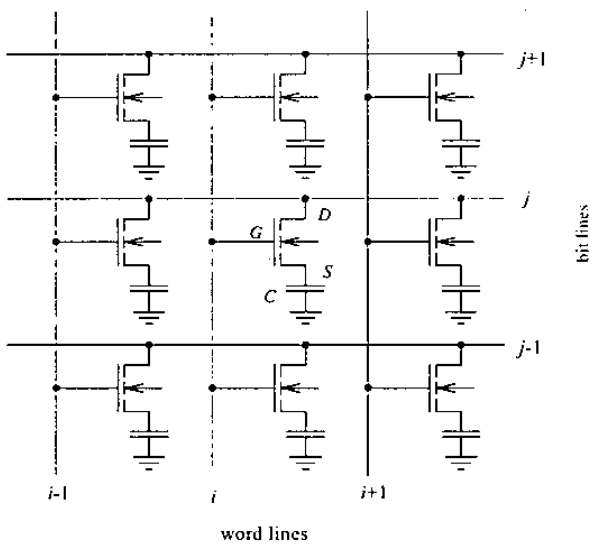

Fig. 2. DRAM architecture utilizing 1T/1C memory cells.

If the memory array shown in Fig. 2 is implemented into $\mathrm{SiC}$, the memory becomes nonvolatile. This is due the 
absence of reverse-bias current of the source-to-substrate $\mathrm{N}-\mathrm{P}$ junction: (1) the diffusion current (the current of the minority carriers) does not exist because there are no minority carriers, and (2) the generation current is negligibly small because of the negligibly low surfacegeneration rate. As opposed to any other type of nonvolatile memory, however, this memory can still be used for data processing. This is because the capacitor charging and discharging through the MOSFET enables infinite number of fast writing cycles. To distinguish this unique type of memory from the other types of nonvolatile memories, we propose the name nonvolatile dynamic memory. The term dynamic stands next to the term volatile, so it makes it obvious that it does not mean a memory that needs to be refreshed, such as the silicon dynamic RAM (DRAM). Taken together with the term nonvolatile, dynamic means that the memory can be used for data processing, something that cannot be achieved with the memories that are just nonvolatile.

The memory cell in the array shown in Fig. 2 was patented in 1995 by Cooper, Palmour, and Carter [34]. The experimental results in the patent are given for a BJT used in place of the MOSFET, because at that time the surface leakage due to surface generation was too high to enable the use of a MOSFET. Even with the BJT, it was found that the retention time of the memory was limited by leakage through the surface that passivates the $\mathrm{P}-\mathrm{N}$ junctions

\section{DISCUSSION}

The conversion of the DRAM array of Fig. 2 into a nonvolatile memory, by implementing it into $\mathrm{SiC}$, provides application opportunities. Nonetheless, this type of nonvolatile dynamic memory does not address a very important limitation of the $1 \mathrm{~T} / 1 \mathrm{C}$ memory cell. This limitation is related to the limited increase of memory capacity. To increase the memory capacity, the cell size has to be decreased, which means the value of the memory capacitance has to be reduced as well. The logic states are memorized as two different values of the capacitance, so the capacitance difference has to be sensed to read the stored information. However, as the capacitance value is decreased it becomes increasingly difficult to sense the difference between the two capacitance levels.

This limitation to the capacity increase is the same for both, the volatile DRAM on $\mathrm{Si}$ and the potential nonvolatile dynamic RAM on SiC. A very important advantage of Si-based DRAM, however, is that it is a well established technology that provides unchallengeable memory capacity for a given price. The implementation of the $1 \mathrm{~T} / 1 \mathrm{C}$ based array in $\mathrm{SiC}$ would provide the technical advantage of the nonvolatile dynamic RAM, but at a high cost. For that cost, it would appear that competitive applications could be achieved by combining volatile DRAM with flash memories.
Therefore, to utilize the unique potential of $\mathrm{SiC}$ with passivated surface for developing nonvolatile dynamic memories, new cells need to be developed that would provide increase in memory capacity beyond the level achievable in silicon technology.

\section{CONCLUSIONS}

$\mathrm{SiC}$ is the second semiconductor that has electronically passivated surface to industrial standards This passivation has been achieved by the nitrided $\mathrm{SiC}-$ $\mathrm{SiO}_{2}$ interface. Given the high-level of development of the first semiconductor with electronically passivated surface, the silicon, the superior bulk properties of $\mathrm{SiC}$ have to be utilized for potential commercial applications. Thanks to the higher breakdown field of $\mathrm{SiC}$, the most obvious application is for power MOSFETs. SiC power MOSFETs exceeding the theoretical figure-of-merit of silicon have been demonstrated. Also, SiC power MOSFETs will be able to work at higher temperatures than silicon. Notwithstanding the commercial success or otherwise of $\mathrm{SiC}$ power MOSFET, the truly remarkable potential for $\mathrm{SiC}$ with passivated surface is for memory applications. This is enabled by the facts that there are no minority carriers in the bulk neutral regions of $\mathrm{SiC}$ and that the surface generation/recombination rates are extremely low in nitrided $\mathrm{SiC}-\mathrm{SiO}_{2}$ interfaces. Importantly, $\mathrm{SiC}$ enables the development of unique type of memories - nomvolatile dynamic memories. There is no other technology or material that can provide this feature. However, without a memory capacity increase, a commercial success of $\mathrm{SiC}$ memories is not certain. With increased memory capacity. however, SiC-based nonvolatile dynamic memories would be in a high-demand application area without any competition.

\section{REFERENCES}

[1] L. Geppert, "The New Indelible Memories", IEEE Spectrum, March 2003 (p. 49).

[2] V.V. Afanas'ev, A. Stesmans, and C.J. Harris, Mater. Sci. Forum Vol. 264-268, p. 857, 1998

[3] R. Schoner, P. Friedrichs, D. Peters, and D. Stephani, IEEE Electron. Dev. Lett. Vol. 20, p. 241, 1999.

[4] H.F. Li, S. Dimitrijev, H.B. Harrison, and D, Swcatman: Appl. Phys. Lett. Vol. 70, p. 2028, 1997

[5] P.T. Lai, J.P. Xu, Y.C. Cheng, IEEE Trans. Electron Dev. Vol. 46, p. 2311, 1999

[6] J.P. Xu, P.T. Lai, C.L. Chan, B. Li, Y.C. Cheng, IEEE Electron Dev. Lett. Vol. 21, p. 298, 2000.

[7] L.A. Lipkin, M.K. Das, J.W. Palmour, Mater. Sci. Fortum Vol. 389-393, p. 985, 2002.

[8] F. Ciobanu, G. Pensl, H. Nagasawa, A. Schoner, S. Dimitrijev, K.Y. Cheong, V.V. Afanas'ev, and G. Wagner, Mater. Sci. Forum Vol. 433-436, p. 551, 2003. 
[9] P. Tanner, S. Dimitrijev, H.F. Li, D. Sweatman, K.E. Prince, and H.B. Harrison, J. Electron. Mater. Vol. 28, p. 109, 1999.

[10] H.F. Li, S. Dimitrijev, D. Sweatman, H.B. Harrison, P. Tanner, and B. Feil, J. Appl. Phys. Vol. 86, p. 4316, 1999.

[11] P. Jamet and S. Dimitrijev, Appl. Phys. Lett. Vol. 79, p. 323, 2001

[12] H.-F. Li, S. Dimitrijev, D. Sweatman, and H.B. Harrison, Microelectron. Reliab. Vol. 40, p. 283, 2000.

[13] G.Y. Chung, C.C. Tin, J.R. Williams, K. McDonald, R.K. Chanana, R.A. Weller, S.T. Pantelides, L.C. Feldman, O.W Holland, M.K. Das, and J.W. Palmour, IEEE Electron Dev. Lett. Vol. 22, p. 176, 2001.

[14] R. Shorner, P. Friedrichs, D. Peters, D. Stephani, S. Dimitrijev, and P. Jamet, Appl. Phys. Lett. Vol. 80, p. 4253 , 2002.

[15] M.K. Das, G.Y. Chung, J.R. Williams, N.S. Saks, L.A. Lipkin, and J.W. Palmour, Mater. Sci. Forum Vol. 389-393, p. 973,2001

[16] C.-Y. Lu, J.A. Cooper, Jr., T. Tsuji, G. Chung, J.R. Williams, K. McDonald, and L.C. Feldman, IEEE Trans. Electron Dev. Vol. 50, p. 1582, 2003.

[17] H.F. Li, S. Dimitrijev, and H.B. Harrison, IEEE Electron Dev. Lett. Vol. 19, p. 279, 1998.

[18] P. Jamet, S. Dimitrijev, and P. Tanner, J. Appl. Phys. Vol. 90, p. 5058, 2001.

[19] K.Y. Cheong, S. Dimitrijev, J. Han, and H.B. Harrison, $J$. Appl. Phys. Vol. 93, p. 5682, 2003.

[20] S. Dimitrijev, K.Y. Cheong, J. Han, and H.B. Harrison, Appl. Phys. Lett. Vol. 80, p. 3421, 2002.
[21] K.Y. Cheong and S. Dimitrijev, IEEE Electron Dev. Lett. Vol. 23, p. 404,2002

[22] P. Jamet, S. Dimitrijev, and P. Tanner, Mater. Sci. Fortum Vol. 353-356, p. 655, 2001

[23] V.V. Afanas'ev, A. Stesmans, F. Ciobanu, G. Pensl, K.Y. Cheong, and S. Dimitrijev, Appl. Phys. Lett. Vol. 82, p. 568 , 2003.

[24] S. Chakraborty, P.T. Lai, P.C.K. Kwok, Microelectron. Reliab. Vol. 42, p. 455, 2002.

[25] K.Y. Cheong, S. Dimitrijev, and J. Han, Mater. Sci. Fornm Vol. 433-436, p. 583, 2003.

[26] S. Dimitrijev, P. Tanner, and H.B. Harrison: Microelectron. Reliab. Vol. 39, p. 441, 1999.

[27] V.V. Afanas'ev, A. Stesmans, M. Bassler, G. Pensl, and M.J. Shulz, Appl. Phys. Lett. Vol. 76, p. 336, 2000.

[28] N.S. Saks, M.G. Ancona, and R.W. Rendell, Appl. Phys. Lett. Vol. 80, p. 3219, 2002.

[29] H.O. Olafsson, F. Allerstam, and E.O. Sveinbjomsson, Mater. Sci. Forum Vol. 389, p. 1005, 2002

[30] K.Y. Cheong, S. Dimitrijev, and J. Han, IEEE Truns. Electron Dev. Vol. 50, p. 1433, 2003.

[31] S. Dimitrijev, H.B. Harrison, P. Tanner, K. Cheong, and J. Han, "Properties of Nitrided Oxides on SiC", in Recent Major Advances in SiC, New York, Berlin: Springer Verlag, 2003.

[32] M.K. Das, to be published in Mater. Sci. Forum, 2004.

[33] D. Stephani, to be published in Mater. Sci. Forlum, 2004

[34] J.A. Cooper, Jr., J.W. Palmour, and C.H. Carter, US Patent No. $5,465,249,1995$. 